Korean J. Math. 20 (2012), No. 1, pp. 1-18

\title{
GENERALIZED CONDITIONAL INTEGRAL TRANSFORMS, CONDITIONAL CONVOLUTIONS AND FIRST VARIATIONS
}

\author{
Bong Jin Kim* And Byoung Soo KIM
}

\begin{abstract}
We study various relationships that exist among generalized conditional integral transform, generalized conditional convolution and generalized first variation for a class of functionals defined on $K[0, T]$, the space of complex-valued continuous functions on $[0, T]$ which vanish at zero.
\end{abstract}

\section{Definitions and preliminaries}

Let $C_{0}[0, T]$ denote one-parameter Wiener space; that is, the space of all $\mathbb{R}$-valued continuous functions $x(t)$ on $[0, T]$ with $x(0)=0$. Let $\mathcal{M}$ denote the class of all Wiener measurable subsets of $C_{0}[0, T]$ and let $m$ denote Wiener measure. $\left(C_{0}[0, T], \mathcal{M}, m\right)$ is a complete measure space and we denote the Wiener integral of a Wiener integrable functional $F$ by

$$
E_{x}[F(x)]=\int_{C_{0}[0, T]} F(x) m(d x) .
$$

Throughout this paper our starting point is the generalized Wiener integral

$$
E_{x}\left[F\left(Z_{h}(x, \cdot)\right)\right]=\int_{C_{0}[0, T]} F\left(Z_{h}(x, \cdot)\right) m(d x),
$$

Received September 20, 2011. Revised January 3, 2012. Accepted January 10, 2012 .

2010 Mathematics Subject Classification: 28C20.

Key words and phrases: conditional Wiener integral, conditional integral transform, conditional convolution.

This work was supported by the Daejin University Research Grants in 2012.

${ }^{*}$ Corresponding author. 
where $Z_{h}$ is the Gaussian process

$$
Z_{h}(x, t)=\int_{0}^{t} h(u) d x(u)
$$

with $h(\neq 0)$ is in $L_{2}[0, T]$, and $\int_{0}^{t} h(u) d x(u)$ denotes the Paley-WienerZygmund stochastic integral. Of course if $h(t) \equiv 1$ on $[0, T]$, then $Z_{h}(x, t)=x(t)$ and so the generalized Wiener integral (1.2) reduces to the Wiener integral (1.1). We will simply refer to the integral (1.2) as a Wiener integral.

The Gaussian process $Z_{h}$ has mean zero and covariance function $E_{x}\left[Z_{h}(x, s) Z_{h}(x, t)\right]=a(\min \{s, t\})$ where $a(t)=\int_{0}^{t} h^{2}(u) d u$. In addition $Z_{h}(x, t)$ is stochastically continuous in $t$ on $[0, T]$.

Let $K=K[0, T]$ be the space of all $\mathbb{C}$-valued continuous functions defined on $[0, T]$ which vanish at $t=0$ and let $\alpha$ and $\beta$ be nonzero complex numbers. In [2], Cameron and Martin defined a Fourier-Wiener transform of functionals defined on $K[0, T]$. In [3], Cameron and Storvick defined a Fourier-Feynman transform of functionals defined on $C_{0}[0, T]$. In a unifying paper [14], Lee defined an integral transform $\mathcal{F}_{\alpha, \beta}$ of analytic functionals on an abstract Wiener space. For certain values of the parameters $\alpha$ and $\beta$ and for certain classes of functionals, the FourierWiener transform, the Fourier-Feynman transform and the Gauss transform are special cases of the integral transform $\mathcal{F}_{\alpha, \beta}$.

In [21], Yeh studied conditional Wiener integrals of functionals defined on $C_{0}[0, T]$. In [8], Chung and Skoug introduced the concept of a conditional Feynman integral, while in [15], Park and Skoug introduced the concept of a conditional Fourier-Feynman transform and a conditional convolution for functionals defined on $C_{0}[0, T]$.

In this paper we establish various relationships that exist among generalized conditional integral transform, generalized conditional convolution and generalized first variation for a class of functionals defined on $K[0, T]$.

We finish this section by stating definitions of integral transform $\mathcal{F}_{\alpha, \beta}$, convolution $(F * G)_{\alpha}$ and first variation $\delta F$ for functionals defined on $K$. The main focus of [11] was to establish various relationships holding among $\mathcal{F}_{\alpha, \beta} F, \mathcal{F}_{\alpha, \beta} G,(F * G)_{\alpha}, \delta F$ and $\delta G$.

Definition 1.1. Let $F$ be a functional defined on $K$. Then the integral transform $\mathcal{F}_{\alpha, \beta} F$ of $F$ is defined by

$$
\mathcal{F}_{\alpha, \beta} F(y) \equiv E_{x}[F(\alpha x+\beta y)], \quad y \in K
$$


if it exists $[5,11,13,15,19]$.

Definition 1.2. Let $F$ and $G$ be functionals defined on $K$. Then the convolution $(F * G)_{\alpha}$ of $F$ and $G$ is defined by

$$
(F * G)_{\alpha}(y) \equiv E_{x}\left[F\left(\frac{y+\alpha x}{\sqrt{2}}\right) G\left(\frac{y-\alpha x}{\sqrt{2}}\right)\right], \quad y \in K
$$

if it exists $[5,10,11,19,20,22]$.

Definition 1.3. Let $F$ be a functional defined on $K$ and let $w \in K$. Then the first variation $\delta F$ of $F$ is defined by

$$
\left.\delta F(y \mid w) \equiv \frac{\partial}{\partial t} F(y+t w)\right|_{t=0}, \quad y \in K
$$

if it exists $[1,4,11,18]$.

\section{Generalized conditional integral transforms and general- ized conditional convolution}

Let $X: C_{0}[0, T] \rightarrow \mathbb{R}$ be a Wiener measurable functional and let $F: C_{0}[0, T] \rightarrow \mathbb{C}$ be a Wiener integrable functional. Then for $\eta \in$ $\mathbb{R}, E[F \| X](\eta)$ denotes the conditional Wiener integral of $F$ given $X$ $[6,8,16,21]$. In [16], Park and Skoug gave a formula for expressing conditional Wiener integrals in terms of ordinary(i.e., non-conditional) Wiener integrals; namely that for $X(x)=x(T)$,

$$
E_{x}[F(x) \| X(x)](\eta)=E_{x}\left[F\left(x(\cdot)-\frac{\dot{T}}{T} x(T)+\dot{\bar{T}} \eta\right)\right] .
$$

Similarly for the condition $X_{h}(x)=Z_{h}(x, T)$, we can get the formula for expressing conditional Wiener integrals,

$$
E_{x}\left[F\left(Z_{h}(x, \cdot) \| X_{h}(x)\right](\eta)=E_{x}\left[F\left(Z_{T, \eta}^{\{h, a\}}(x, \cdot)\right)\right],\right.
$$

where $Z_{T, \eta}^{\{h, a\}}(x, \cdot)=Z_{h}(x, \cdot)-\frac{a(\cdot)}{a(T)} Z_{h}(x, T)+\frac{a(\cdot)}{a(T)} \eta$.

In this paper we will always condition by

$$
X_{h}(x)=Z_{h}(x, T),
$$

where $Z_{h}$ is given by (1.3).

Definition 2.1. For $F: K[0, T] \rightarrow \mathbb{C}$ we define generalized conditional integral transform, $\mathcal{F}_{\alpha, \beta}\left(F \| X_{h}\right)(y, \eta)$ of $F$ given $X_{h}$ by the formula

$$
\mathcal{F}_{\alpha, \beta}\left(F \| X_{h}\right)(y, \eta)=E_{x}\left[F\left(\alpha Z_{h}(x, \cdot)+\beta y\right) \| X_{h}(x)=\eta\right]
$$


for $y \in K$ and $\eta \in \mathbb{R}$ if it exists.

Definition 2.2. For functionals $F$ and $G$ defined on $K$, we define generalized conditional convolution, $\left((F * G)_{\alpha} \| X_{h}\right)(y, \eta)$, of $(F * G)_{\alpha}$ given $X_{h}$ by the formula

$\left((F * G)_{\alpha} \| X_{h}\right)(y, \eta)=E_{x}\left[F\left(\frac{y+\alpha Z_{h}(x, \cdot)}{\sqrt{2}}\right) G\left(\frac{y-\alpha Z_{h}(x, \cdot)}{\sqrt{2}}\right) \| X_{h}(x)=\eta\right]$

for $y \in K$ and $\eta \in \mathbb{R}$ if it exists.

Next we give a definition of generalized first variation $\delta_{h_{1}, h_{2}} F$ of a functional $F$ on $K$.

Definition 2.3. Let $F$ be a functional defined on $K$ and let $w \in K$ and $h_{1}, h_{2} \in L_{2}[0, T]$. Then the generalized first variation $\delta_{h_{1}, h_{2}} F$ of $F$ is defined by

$$
\delta_{h_{1}, h_{2}} F(y \mid w)=\left.\frac{\partial}{\partial r} F\left(Z_{h_{1}}(y, \cdot)+r Z_{h_{2}}(w, \cdot)\right)\right|_{r=0}
$$

for $y \in K$ if it exists.

REMARK 2.4. (i) When $h \equiv 1$ on $[0, T]$, the generalized conditional integral transform and generalized conditional convolution are reduced to conditional integral transform and conditional convolution, respectively, which are defined and studied in [12].

(ii) When $h_{1}=h_{2} \equiv 1$ on $[0, T]$, our definition of the generalized first variation is reduced to the first variation studied in $[1,4,11,12,18]$.

(iii) Using the formula for expressing conditional Wiener integral with conditioning function $X_{h}(x)=Z_{h}(x, T)$ in [17], we have

$$
\mathcal{F}_{\alpha, \beta}\left(F \| X_{h}\right)(y, \eta)=E_{x}\left[F\left(\alpha Z_{T, \eta}^{\{h, a\}}(x, \cdot)+\beta y(\cdot)\right)\right]
$$

and

$$
\begin{aligned}
& \left((F * G)_{\alpha} \| X_{h}\right)(y, \eta) \\
& =E_{x}\left[F\left(\frac{1}{\sqrt{2}}\left(y(\cdot)+\alpha Z_{T, \eta}^{\{h, a\}}(x, \cdot)\right)\right) G\left(\frac{1}{\sqrt{2}}\left(y(\cdot)-\alpha Z_{T, \eta}^{\{h, a\}}(x, \cdot)\right)\right)\right]
\end{aligned}
$$

where in (2.6) and (2.7) the existence of either side implies the other side and their equality. 
Under rather mild conditions on $F$ and $G$, our first theorem shows that the generalized conditional integral transform of the generalized conditional convolution is the product of generalized conditional integral transforms.

Theorem 2.5. Let $\alpha$ and $\beta$ be nonzero complex numbers. Assume that for $F: K \rightarrow \mathbb{C}$ and $G: K \rightarrow \mathbb{C}, \mathcal{F}_{\alpha, \beta}\left(\left((F * G)_{\alpha} \| X_{h}\right)\left(\cdot, \eta_{1}\right) \| X_{h}\right)$, $\mathcal{F}_{\alpha, \beta}\left(F \| X_{h}\right)$ and $\mathcal{F}_{\alpha, \beta}\left(G \| X_{h}\right)$ all exist for a.e. $\eta_{1} \in \mathbb{R}$. Then

$$
\begin{aligned}
& \mathcal{F}_{\alpha, \beta}\left(\left((F * G)_{\alpha} \| X_{h}\right)\left(\cdot, \eta_{1}\right) \| X_{h}\right)\left(y, \eta_{2}\right) \\
& =\mathcal{F}_{\alpha, \beta}\left(F \| X_{h}\right)\left(\frac{y}{\sqrt{2}}, \frac{\eta_{2}+\eta_{1}}{\sqrt{2}}\right) \mathcal{F}_{\alpha, \beta}\left(G \| X_{h}\right)\left(\frac{y}{\sqrt{2}}, \frac{\eta_{2}-\eta_{1}}{\sqrt{2}}\right)
\end{aligned}
$$

for all $y \in K$ and a.e. $\eta_{2} \in \mathbb{R}$.

Proof. From equations (2.3) through (2.7) we have the following;

$$
\begin{aligned}
R \equiv \mathcal{F}_{\alpha, \beta}\left(\left((F * G)_{\alpha} \| X_{h}\right)\left(\cdot, \eta_{1}\right) \| X_{h}\right)\left(y, \eta_{2}\right) \\
=E_{x}\left[E _ { w } \left[F\left(\frac{\beta y(\cdot)}{\sqrt{2}}+\frac{\alpha}{\sqrt{2}} Z_{T, \eta_{2}+\eta_{1}}^{\{h, a\}}(x+w, \cdot)\right)\right.\right. \\
\left.\left.\cdot G\left(\frac{\beta y(\cdot)}{\sqrt{2}}+\frac{\alpha}{\sqrt{2}} Z_{T, \eta_{2}-\eta_{1}}^{\{h, a\}}(x-w, \cdot)\right)\right]\right] .
\end{aligned}
$$

Since $Z_{h}(x+w, \cdot)-\frac{a(\cdot)}{a(T)} Z_{h}(x+w, T)$ and $Z_{h}(x-w, \cdot)-\frac{a(\cdot)}{a(T)} Z_{h}(x-w, T)$ are independent processes as can be seen by checking their covariance function, we can see that

$$
\begin{aligned}
R= & E_{x}\left[E_{w}\left[F\left(\frac{\beta y(\cdot)}{\sqrt{2}}+\frac{\alpha}{\sqrt{2}} Z_{T, \eta_{2}+\eta_{1}}^{\{h, a\}}(x+w, \cdot)\right)\right]\right] \\
& \cdot E_{x}\left[E_{w}\left[G\left(\frac{\beta y(\cdot)}{\sqrt{2}}+\frac{\alpha}{\sqrt{2}} Z_{T, \eta_{2}-\eta_{1}}^{\{h, a\}}(x-w, \cdot)\right)\right]\right]
\end{aligned}
$$

Also the processes $\frac{Z_{h}(x+w, \cdot)}{\sqrt{2}}$ and $\frac{Z_{h}(x-w, \cdot)}{\sqrt{2}}$ are each equivalent to the process $Z_{h}(x, \cdot)$, and the equation (2.6) give us the following result;

$$
\begin{aligned}
R & =E_{x}\left[F\left(\frac{\beta y(\cdot)}{\sqrt{2}}+\alpha Z_{T, \frac{\eta_{2}+\eta_{1}}{\sqrt{2}}}^{\{h, y}(x)\right)\right] E_{x}\left[G \left(\frac{\beta y(\cdot)}{\sqrt{2}}+\alpha Z_{T, \frac{\eta_{2}-\eta_{1}}{\sqrt{2}}}^{\{h, x, \cdot))]}\right.\right. \\
& =\mathcal{F}_{\alpha, \beta}\left(F \| X_{h}\right)\left(\frac{y}{\sqrt{2}}, \frac{\eta_{2}+\eta_{1}}{\sqrt{2}}\right) \mathcal{F}_{\alpha, \beta}\left(G \| X_{h}\right)\left(\frac{y}{\sqrt{2}}, \frac{\eta_{2}-\eta_{1}}{\sqrt{2}}\right)
\end{aligned}
$$

which completes the proof. 
Next we describe the class of functionals that we work with in this paper. Let $\left\{\theta_{1}, \theta_{2}, \ldots\right\}$ be a complete orthonormal set of $\mathbb{R}$-valued functions in $L_{2}[0, T]$. Furthermore assume that each $\theta_{j}$ is of bounded variation on $[0, T]$. Then for each $y \in K$ and $j \in\{1,2, \ldots\}$, the Riemann-Stieltjes integral $\left\langle\theta_{j}, y\right\rangle \equiv \int_{0}^{T} \theta_{j}(t) d y(t)$ exists. Furthermore

$$
\left|\left\langle\theta_{j}, y\right\rangle\right|=\left|\theta_{j}(T) y(T)-\int_{0}^{T} y(t) d \theta_{j}(t)\right| \leq M_{j}\|y\|_{\infty}
$$

with

$$
M_{j}=\left|\theta_{j}(T)\right|+\operatorname{Var}\left(\theta_{j},[0, T]\right) .
$$

For $0 \leq \sigma<1$, let $E_{\sigma}$ be the space of all functionals $F: K \rightarrow \mathbb{C}$ of the form

$$
F(y)=f\left(\left\langle\theta_{1}, y\right\rangle, \ldots,\left\langle\theta_{n}, y\right\rangle\right)=f(\langle\vec{\theta}, y\rangle)
$$

for some positive integer $n$, where $f\left(\lambda_{1}, \ldots, \lambda_{n}\right)=f(\vec{\lambda})$ is an entire function of $n$ complex variables $\lambda_{1}, \ldots, \lambda_{n}$ of exponential type; that is to say

$$
|f(\vec{\lambda})| \leq A_{F} \exp \left\{B_{F} \sum_{j=1}^{n}\left|\lambda_{j}\right|^{1+\sigma}\right\}
$$

for some positive constants $A_{F}$ and $B_{F}$.

In [12], the current authors and Skoug showed that for all $F$ and $G$ in $E_{\sigma}, \mathcal{F}_{\alpha, \beta}(F \| X)$ and $\left((F * G)_{\alpha} \| X\right)$ exist and belong to $E_{\sigma}$ for all nonzero complex numbers $\alpha$ and $\beta$ and the condition by $X(x)=x(T)$ while $\delta F(y \mid w)$ exists and belongs to $E_{\sigma}$ for all $y$ and $w$ in $K$.

For $F$ of the form (2.11), as we will see below in Theorem 2.7, when we evaluate the generalized conditional integral transform of $F$ given $X_{h}$, we encounter the Riemann-Stieltjes integrals $\left\langle\theta_{j}(\cdot), Z_{T, \eta}^{\{h, a\}}(x, \cdot)\right\rangle$. Letting

$$
b_{j}=\frac{1}{a(T)} \int_{0}^{T} \theta_{j}(t) d a(t)
$$

we see that

$$
\left\langle\theta_{j}(\cdot), Z_{T, \eta}^{\{h, a\}}(x, \cdot)\right\rangle=\left\langle\theta_{j}(\cdot)-b_{j}, Z_{h}(x, \cdot)\right\rangle+\eta b_{j}
$$

for $x \in C_{0}[0, T]$. 
Take a $n \times n$ matrix $C=\left(c_{j, k}\right)$ and an orthonormal set $\left\{\phi_{1}, \ldots, \phi_{n}\right\}$ on $[0, T]$ satisfying

$$
\vec{\theta}-\vec{b}=\vec{\phi} C .
$$

For details on the matrix $C$ and the orthonormal set $\left\{\phi_{1}, \ldots, \phi_{n}\right\}$, see Section 2 of [12].

The following lemma [7], which follows quite easily from the definition of the Paley-Wiener-Zygmund stochastic integral, (2.14) and (2.15), plays a key role in the proof of Theorem 2.7. In view of the following lemma, throughout this paper we require $h$ to be in $L_{\infty}[0, T]$ with $\left\{\phi_{1} h, \ldots, \phi_{n} h\right\}$ be an orthogonal set in $L_{2}[0, T]$ rather than simply in $L_{2}[0, T]$.

Lemma 2.6. For each $\phi \in L_{2}[0, T]$ and each $h \in L_{\infty}[0, T]$,

$$
\int_{0}^{T} \phi(t) d Z_{h}(x, t)=\int_{0}^{T} \phi(t) h(t) d x(t)
$$

for $s$-a.e. $x \in C_{0}[0, T]$, that is, $\left\langle\phi, Z_{h}(x, \cdot)\right\rangle=\langle\phi h, x\rangle$.

TheOREM 2.7. Let $F \in E_{\sigma}$ be given by (2.11), $h \in L_{\infty}[0, T]$ with $\left\|\phi_{j} h\right\|_{2}^{2}>0$ for $j=1,2, \cdots n$, and let $X_{h}$ be given by (2.2). Then the generalized conditional integral transform $\mathcal{F}_{\alpha, \beta}\left(F \| X_{h}\right)(y, \eta)$ exists, belongs to $E_{\sigma}$ and is given by the formula

$$
\mathcal{F}_{\alpha, \beta}\left(F \| X_{h}\right)(y, \eta)=K_{h}(\eta ;\langle\vec{\theta}, y\rangle)
$$

for all $y \in K$ and a.e. $\eta \in \mathbb{R}$, where

$$
\begin{aligned}
& K_{h}(\eta ; \vec{\lambda}) \\
& =\left((2 \pi)^{n} \prod_{j=1}^{n}\left\|\phi_{j} h\right\|_{2}^{2}\right)^{-\frac{1}{2}} \int_{\mathbb{R}^{n}} f(\alpha \vec{u} C+\alpha \eta \vec{b}+\beta \vec{\lambda}) \exp \left\{-\frac{1}{2} \sum_{j=1}^{n} \frac{u_{j}^{2}}{\left\|\phi_{j} h\right\|_{2}^{2}}\right\} d \vec{u} .
\end{aligned}
$$

Proof. For each $y \in K$ and a.e. $\eta \in \mathbb{R}$,

$$
\mathcal{F}_{\alpha, \beta}\left(F \| X_{h}\right)(y, \eta)=E_{x}\left[f\left(\alpha\left\langle\vec{\theta}, Z_{T, \eta}^{\{h, a\}}(x, \cdot)\right\rangle+\beta\langle\vec{\theta}, y\rangle\right)\right] .
$$

Using (2.14) and (2.15), we have

$$
\begin{aligned}
\mathcal{F}_{\alpha, \beta}\left(F \| X_{h}\right)(y, \eta) & =E_{x}\left[f\left(\alpha\left\langle\vec{\theta}-\vec{b}, Z_{h}(x, \cdot)\right\rangle+\alpha \eta \vec{b}+\beta\langle\vec{\theta}, y\rangle\right)\right] \\
& =E_{x}\left[f\left(\alpha\left\langle\vec{\phi}, Z_{h}(x, \cdot)\right\rangle C+\alpha \eta \vec{b}+\beta\langle\vec{\theta}, y\rangle\right)\right] .
\end{aligned}
$$


By Lemma 2.6 and a well-known Wiener integration theorem, we see that the last expression is equal to $K_{h}(\eta ;\langle\vec{\theta}, y\rangle)$, where $K_{h}(\eta ; \cdot)$ is given by (2.18). By [9, Theorem 3.15] $K_{h}(\eta ; \vec{\lambda})$ is an entire function. Moreover by the inequality $(2.12)$ we have

$$
\begin{aligned}
& \left|K_{h}(\eta ; \vec{\lambda})\right| \\
\leq & \left((2 \pi)^{n} \prod_{j=1}^{n}\left\|\phi_{j} h\right\|_{2}^{2}\right)^{-1 / 2} A_{F} \exp \left\{B_{F}(3|\beta|)^{1+\sigma} \sum_{j=1}^{n}\left|\lambda_{j}\right|^{1+\sigma}\right\} \\
& \cdot \int_{\mathbb{R}^{n}} \exp \left\{B_{F}(3|\alpha|)^{1+\sigma} \sum_{j=1}^{n}\left(\left|(\vec{u} C)_{j}\right|^{1+\sigma}+\left|\eta b_{j}\right|^{1+\sigma}\right)-\frac{1}{2} \sum_{j=1}^{n} \frac{u_{j}^{2}}{\left\|\phi_{j} h\right\|_{2}^{2}}\right\} d \vec{u} \\
= & A_{\mathcal{F}_{\alpha, \beta ; h} F} \exp \left\{B_{\mathcal{F}_{\alpha, \beta ; h} F} \sum_{j=1}^{n}\left|\lambda_{j}\right|^{1+\sigma}\right\}
\end{aligned}
$$

where $B_{\mathcal{F}_{\alpha, \beta ; h} F}=B_{F}(3|\beta|)^{1+\sigma}$, and

$$
\begin{aligned}
A_{\mathcal{F}_{\alpha, \beta ; h} F}= & A_{F}\left((2 \pi)^{n} \prod_{j=1}^{n}\left\|\phi_{j} h\right\|_{2}^{2}\right)^{-1 / 2} \int_{\mathbb{R}^{n}} \exp \left\{B_{F}(3|\alpha|)^{1+\sigma}\right. \\
& \left.\sum_{j=1}^{n}\left(\left|(\vec{u} C)_{j}\right|^{1+\sigma}+\left|\eta b_{j}\right|^{1+\sigma}\right)-\frac{1}{2} \sum_{j=1}^{n} \frac{u_{j}^{2}}{\left\|\phi_{j} h\right\|_{2}^{2}}\right\} d \vec{u}<\infty .
\end{aligned}
$$

Hence $\mathcal{F}_{\alpha, \beta}\left(F \| X_{h}\right)(y, \eta) \in E_{\sigma}$ as a function of $y$.

REMARK 2.8. For any $F \in E_{\sigma}$ and $G \in E_{\sigma}$ we can always express $F$ by equation (2.11) and $G$ by

$$
G(y)=g\left(\left\langle\theta_{1}, y\right\rangle, \cdots,\left\langle\theta_{n}, y\right\rangle\right)
$$

using the same positive integer $n$.

In our next theorem we show that the generalized conditional convolution of functionals from $E_{\sigma}$ is an element of $E_{\sigma}$.

Theorem 2.9. Let $F, G \in E_{\sigma}$ be given by (2.11) and (2.20) with corresponding entire functions $f$ and $g$, respectively. And let $X_{h}$ be given by (2.2) with $h \in L_{\infty}[0, T]$ and $\left\|\phi_{j} h\right\|_{2}^{2}>0$ for $j=1, \cdots, n$. Then the generalized conditional convolution $\left((F * G)_{\alpha} \| X_{h}\right)(y, \eta)$ exists for all $y \in K$ and a.e. $\eta \in \mathbb{R}$, belongs to $E_{\sigma}$, and is given by the formula

$$
\left((F * G)_{\alpha} \| X_{h}\right)(y, \eta)=L_{h}(\eta ;\langle\vec{\theta}, y\rangle)
$$


where

$$
\begin{aligned}
& L_{h}(\eta ; \vec{\lambda}) \\
& =\left((2 \pi)^{n} \prod_{j=1}^{n}\left\|\phi_{j} h\right\|_{2}^{2}\right)^{-1 / 2} \int_{\mathbb{R}^{n}} f\left(\frac{\vec{\lambda}+\alpha \vec{u} C+\alpha \eta \vec{b}}{\sqrt{2}}\right) g\left(\frac{\vec{\lambda}-\alpha \vec{u} C-\alpha \eta \vec{b}}{\sqrt{2}}\right) \\
& \quad \exp \left\{-\frac{1}{2} \sum_{j=1}^{n} \frac{u_{j}^{2}}{\left\|\phi_{j} h\right\|_{2}^{2}}\right\} d \vec{u} .
\end{aligned}
$$

Proof. For each $y \in K$ and a.e. $\eta \in \mathbb{R}$,

$$
\begin{aligned}
L \equiv & \left((F * G)_{\alpha} \| X_{h}\right)(y, \eta) \\
= & E_{x}\left[f\left(\frac{1}{\sqrt{2}}\left(\langle\vec{\theta}, y\rangle+\alpha\left\langle\vec{\theta}, Z_{h}(x, \cdot)-\frac{a(\cdot)}{a(T)} Z_{h}(x, T)+\frac{a(\cdot)}{a(T)} \eta\right\rangle\right)\right)\right. \\
& \left.g\left(\frac{1}{\sqrt{2}}\left(\langle\vec{\theta}, y\rangle-\alpha\left\langle\vec{\theta}, Z_{h}(x, \cdot)-\frac{a(\cdot)}{a(T)} Z_{h}(x, T)+\frac{a(\cdot)}{a(T)} \eta\right\rangle\right)\right)\right] .
\end{aligned}
$$

By (2.14), (2.15) and a well-known Wiener integration theorem, we see that the last expression is equal to $L_{h}(\eta ;\langle\vec{\theta}, y\rangle)$ where $L_{h}(\eta ; \vec{\lambda})$ is given by (2.22). By [9, Theorem 3.15], $L_{h}(\eta ; \vec{\lambda})$ is an entire function and

$$
\begin{aligned}
& \left|L_{h}(\eta ; \vec{\lambda})\right| \\
& \leq\left((2 \pi)^{n} \prod_{j=1}^{n}\left\|\phi_{j} h\right\|_{2}^{2}\right)^{-\frac{1}{2}} A_{F} A_{G} \exp \left\{\left(B_{F}+B_{G}\right)\left(\frac{3}{\sqrt{2}}\right)^{1+\sigma} \sum_{j=1}^{n}\left|\lambda_{j}\right|^{1+\sigma}\right\} \\
& \cdot \int_{\mathbb{R}^{n}} \exp \left\{\left(B_{F}+B_{G}\right)\left(\frac{3|\alpha|}{\sqrt{2}}\right)^{1+\sigma} \sum_{j=1}^{n}\left(\left|(\vec{u} C)_{j}\right|^{1+\sigma}+\left|\eta b_{j}\right|^{1+\sigma}-\frac{1}{2} \frac{u_{j}^{2}}{\left\|\phi_{j} h\right\|_{2}^{2}}\right)\right\} d \vec{u} \\
& =A_{(F * G)_{\alpha} ; h} \exp \left\{B_{(F * G)_{\alpha} ; h} \sum_{j=1}^{n}\left|\lambda_{j}\right|^{1+\sigma}\right\},
\end{aligned}
$$

where

$$
B_{(F * G)_{\alpha} ; h}=\left(B_{F}+B_{G}\right)\left(\frac{3}{\sqrt{2}}\right)^{1+\sigma}
$$


and

$$
\begin{aligned}
A_{(F * G)_{\alpha} ; h}= & A_{F} A_{G}\left((2 \pi)^{n} \prod_{j=1}^{n}\left\|\phi_{j} h\right\|_{2}^{2}\right)^{-\frac{1}{2}} \int_{\mathbb{R}^{n}} \exp \left\{\left(B_{F}+B_{G}\right)\left(\frac{3|\alpha|}{\sqrt{2}}\right)^{1+\sigma}\right. \\
& \left.\sum_{j=1}^{n}\left(\left|(\vec{u} C)_{j}\right|^{1+\sigma}+\left|\eta b_{j}\right|^{1+\sigma}\right)-\frac{1}{2} \sum_{j=1}^{n} \frac{u_{j}^{2}}{\left\|\phi_{j} h\right\|_{2}^{2}}\right\} d \vec{u}<\infty .
\end{aligned}
$$

Hence $\left((F * G)_{\alpha} \| X_{h}\right)(y, \eta) \in E_{\sigma}$ as a function of $y$.

REMARK 2.10. Unlike the convolution product, the generalized conditional convolution product is not commutative because $\left((F * G)_{\alpha} \| X_{h}\right)(y, \eta)=$ $\left((G * F)_{\alpha} \| X_{h}\right)(y,-\eta)$. However the usual additive distribution properties hold for the generalized conditional convolution product.

As in [12, Theorem 2.6], we can show that the generalized first variation $\delta_{h_{1}, h_{2}} F(y \mid w)$ of functionals $F$ in $E_{\sigma}$ is an element of $E_{\sigma}$, both as a function of $y$ for fixed $w$ and as a function of $w$ for fixed $y$.

Theorem 2.11. Let $F \in E_{\sigma}$ be given by (2.11) and let $h_{1}$ and $h_{2}$ be in $L_{\infty}[0, T]$. Then for all $y$ and $w$ in $K$,

$$
\begin{aligned}
\delta_{h_{1}, h_{2}} F(y \mid w) & =\sum_{j=1}^{n}\left\langle\theta_{j}, Z_{h_{2}}(w, \cdot)\right\rangle f_{j}\left(\left\langle\vec{\theta}, Z_{h_{1}}(y, \cdot)\right\rangle\right) \\
& =\sum_{j=1}^{n}\left\langle\theta_{j}, Z_{h_{2}}(w, \cdot)\right\rangle F_{j}\left(Z_{h_{1}}(y, \cdot)\right)
\end{aligned}
$$

where $f_{j}(\vec{\lambda})=\frac{\partial}{\partial \lambda_{j}} f\left(\lambda_{1}, \ldots, \lambda_{n}\right)$ and $F_{j}(\cdot)=f_{j}(\langle\vec{\theta}, \cdot\rangle)$. In addition, as a function of $y, \delta_{h_{1}, h_{2}} F(y \mid w)$ is an element of $E_{\sigma}$ with $B_{\delta_{h_{1}, h_{2}} F(\cdot \mid w)}=$ $2^{1+\sigma} B_{F}$ and with

$$
A_{\delta_{h_{1}, h_{2}} F(\cdot \mid w)}=A_{F} \exp \left\{2^{1+\sigma} B_{F}\right\}\left(\|w\|_{\infty} \sum_{j=1}^{n} N_{j}\right)
$$

where $\left|\left\langle\theta_{j} h_{2}, w\right\rangle\right| \leq N_{j}\|w\|_{\infty}$, and $N_{j}=\left|\theta_{j}(T) h_{2}(T)\right|+\operatorname{Var}\left(\theta_{j} h_{2},[0, T]\right)$. Furthermore, as a function of $w, \delta_{h_{1}, h_{2}} F(y \mid w)$ is an element of $E_{\sigma}$ with $B_{\delta_{h_{1}, h_{2}} F(y \mid \cdot)}=1$ and with

$$
A_{\delta_{h_{1}, h_{2}} F(y \mid \cdot)}=n e^{-\frac{1}{2}} A_{F} \exp \left\{2^{1+\sigma} B_{F}\left(1+\sum_{j=1}^{n}\left(\left\|h_{1}\right\|_{\infty}\|y\|_{\infty} M_{j}\right)^{1+\sigma}\right)\right\} .
$$


REMARK 2.12. Note that in view of theorems 2.7, 2.9, and 2.11 above, all of the functionals that arise in Section 3 below are automatically elements of $E_{\sigma}$.

\section{Various relationships involving the concepts}

In this section we establish the various relationships involving the three concepts of generalized conditional integral transform, generalized conditional convolution and generalized first variation for functionals belonging to $E_{\sigma}$. These various relationships, as well as alternative expressions for some of them, are given by formula (2.8) above, formulas (3.4) through (3.6), (3.8) and (3.10) through (3.12) below. It is interesting to note that the left hand side of each of these formulas involve exactly two of the three concepts, while each right hand side involves at most one of these concepts.

The following lemma plays a key role in establishing several formulas throughout this section. 0 ,

Lemma 3.1. For all $j \in\{1,2, \ldots, n\}$ and $h \in L_{\infty}[0, T]$ with $\left\|\phi_{j} h\right\|_{2}^{2}>$

$$
E_{x}\left[\left\langle\theta_{j}-b_{j}, Z_{h}(x, \cdot)\right\rangle\right]=0,
$$

while for all $j$ and $l$ in $\{1,2, \ldots, n\}$,

$$
E_{x}\left[\left\langle\theta_{j}-b_{j}, Z_{h}(x, \cdot)\right\rangle\left\langle\theta_{l}-b_{l}, Z_{h}(x, \cdot)\right\rangle\right]=D_{j, l ; h}
$$

where

$$
D_{j, l ; h}=\sum_{k=1}^{n} c_{k, j} c_{k, l}\left\|\phi_{k} h\right\|_{2}^{2}
$$

Proof. By equation (2.15), $\theta_{j}-b_{j}=\sum_{k=1}^{n} c_{k, j} \phi_{k}$ for all $j \in\{1,2, \ldots, n\}$, and hence

$$
E_{x}\left[\left\langle\theta_{j}-b_{j}, Z_{h}(x, \cdot)\right\rangle\right]=\sum_{k=1}^{n} c_{k, j} E_{x}\left[\left\langle\phi_{k}, Z_{h}(x, \cdot)\right\rangle\right] .
$$

But by Lemma 2.6,

$$
E_{x}\left[\left\langle\phi_{k}, Z_{h}(x, \cdot)\right\rangle\right]=\left(2 \pi\left\|\phi_{k} h\right\|_{2}^{2}\right)^{-1 / 2} \int_{\mathbb{R}} u \exp \left\{-\frac{1}{2} \frac{u^{2}}{\left\|\phi_{k} h\right\|_{2}^{2}}\right\} d u=0
$$


and so we obtain (3.1).

Similarly for all $j$ and $l$ in $\{1,2, \cdots, n\}$,

$$
\begin{aligned}
& E_{x}\left[\left\langle\theta_{j}-b_{j}, Z_{h}(x, \cdot)\right\rangle\left\langle\theta_{l}-b_{l}, Z_{h}(x, \cdot)\right\rangle\right] \\
& =\sum_{k=1}^{n} \sum_{m=1}^{n} c_{k, j} c_{m, l} E_{x}\left[\left\langle\phi_{k}, Z_{h}(x, \cdot)\right\rangle\left\langle\phi_{m}, Z_{h}(x, \cdot)\right\rangle\right] \\
& =\sum_{k=1}^{n} c_{k, j} c_{k, l}\left\|\phi_{k} h\right\|_{2}^{2}=D_{j, l ; h}
\end{aligned}
$$

as desired, because for $k \neq m$,

$$
E_{x}\left[\left\langle\phi_{k}, Z_{h}(x, \cdot)\right\rangle\left\langle\phi_{m}, Z_{h}(x, \cdot)\right\rangle\right]=E_{x}\left[\left\langle\phi_{k} h, x\right\rangle\left\langle\phi_{m} h, x\right\rangle\right]=0
$$

and this completes the proof.

Our first formula (2.8) is useful because it allows us to calculate $\mathcal{F}_{\alpha, \beta}\left(\left((F * G)_{\alpha} \| X_{h}\right)\left(\cdot, \eta_{1}\right) \| X_{h}\right)\left(y, \eta_{2}\right)$ without ever actually calculating $(F * G)_{\alpha}$ or $\left((F * G)_{\alpha} \| X_{h}\right)$.

Theorem 3.2. Let $F$ and $G$ be as in Theorem 2.9. Then equation (2.8) holds for all $y \in K$ and a.e. $\eta_{1}, \eta_{2} \in \mathbb{R}$.

Proof. The left hand side of (2.8) exists by Theorem 2.9 and Theorem 2.7, while the right hand side of equation (2.8) exists by Theorem 2.7. The equality in equation (2.8) then follows from Theorem 2.5.

Our next formula (3.4), giving the conditional convolution of conditional integral transforms, follows from Theorem 2.7, Theorem 2.9 and a well-known Wiener integration formula.

Theorem 3.3. Let $F$ and $G$ be as in Theorem 2.9. Then for all $y \in K$ and a.e. $\eta_{1}, \eta_{2}, \eta_{3} \in \mathbb{R}$,

$$
\begin{gathered}
\left(\left(\mathcal{F}_{\alpha, \beta}\left(F \| X_{h}\right)\left(\cdot, \eta_{1}\right) * \mathcal{F}_{\alpha, \beta}\left(G \| X_{h}\right)\left(\cdot, \eta_{2}\right)\right)_{\alpha} \| X_{h}\right)\left(y, \eta_{3}\right) \\
=\left[(2 \pi)^{n} \prod_{j=1}^{n}\left\|\phi_{j} h\right\|_{2}^{2}\right]^{-\frac{3}{2}} \int_{\mathbb{R}^{3 n}} f\left(\alpha \vec{v} C+\alpha \eta_{1} \vec{b}+\frac{\beta}{\sqrt{2}}\left(\langle\vec{\theta}, y\rangle+\alpha \vec{u} C+\alpha \eta_{3} \vec{b}\right)\right) \\
g\left(\alpha \vec{w} C+\alpha \eta_{2} \vec{b}+\frac{\beta}{\sqrt{2}}\left(\langle\vec{\theta}, y\rangle-\alpha \vec{u} C-\alpha \eta_{3} \vec{b}\right)\right) \\
\exp \left\{-\frac{1}{2} \sum_{j=1}^{n} \frac{1}{\left\|\phi_{j} h\right\|_{2}^{2}}\left(u_{j}^{2}+v_{j}^{2}+w_{j}^{2}\right)\right\} d \vec{u} d \vec{v} d \vec{w} .
\end{gathered}
$$


In our next theorem we obtain a formula for the generalized first variation of the conditional convolution of functionals from $E_{\sigma}$.

Theorem 3.4. Let $F \in E_{\sigma}$ be given by (2.11) and let $h, h_{1}$ and $h_{2}$ be in $L_{\infty}[0, T]$. Then for a.e. $\eta \in \mathbb{R}$,

$$
\begin{aligned}
& \delta_{h_{1}, h_{2}}\left((F * G)_{\alpha} \| X_{h}\right)(\cdot, \eta)(y \mid w) \\
& =\sum_{j=1}^{n} \frac{\left\langle\theta_{j}, Z_{h_{2}}(w, \cdot)\right\rangle}{\sqrt{2}}\left[\left(\left(F_{j} * G\right)_{\alpha} \| X_{h}\right)\left(Z_{h_{1}}(y, \cdot), \eta\right)\right. \\
& \left.\quad+\left(\left(F * G_{j}\right)_{\alpha} \| X_{h}\right)\left(Z_{h_{1}}(y, \cdot), \eta\right)\right]
\end{aligned}
$$

for all $y$ and $w$ in $K$.

Proof. By the definition of the generalized first variation and (2.21) it follows that

$$
\begin{aligned}
A \equiv & \delta_{h_{1}, h_{2}}\left((F * G)_{\alpha} \| X_{h}\right)(\cdot, \eta)(y \mid w) \\
= & \left.\frac{\partial}{\partial r}\left((F * G)_{\alpha} \| X_{h}\right)\left(Z_{h_{1}}(y, \cdot)+r Z_{h_{2}}(w, \cdot), \eta\right)\right|_{r=0} \\
= & \frac{\partial}{\partial r} E_{x}\left[f\left(\frac{1}{\sqrt{2}}\left(\left\langle\vec{\theta}, Z_{h_{1}}(y, \cdot)+r Z_{h_{2}}(w, \cdot)\right\rangle+\alpha\left\langle\vec{\phi}, Z_{h}(x, \cdot)\right\rangle C+\alpha \eta \vec{b}\right)\right)\right. \\
& \left.\quad g\left(\frac{1}{\sqrt{2}}\left[\left\langle\vec{\theta}, Z_{h_{1}}(y, \cdot)+r Z_{h_{2}}(w, \cdot)\right\rangle-\alpha\left\langle\vec{\phi}, Z_{h}(x, \cdot)\right\rangle C-\alpha \eta \vec{b}\right)\right)\right]\left.\right|_{r=0} .
\end{aligned}
$$

Evaluating partial derivative in the last expression we obtain that

$$
\begin{aligned}
A=\sum_{j=1}^{n} \frac{\left\langle\theta_{j}, Z_{h_{2}}(w, \cdot)\right\rangle}{\sqrt{2}} E_{x}\left[f_{j}\left(\frac{1}{\sqrt{2}}\left(\left\langle\vec{\theta}, Z_{h_{1}}(y, \cdot)\right\rangle+\alpha\left\langle\vec{\phi}, Z_{h}(x, \cdot)\right\rangle C+\alpha \eta \vec{b}\right)\right)\right. \\
g\left(\frac{1}{\sqrt{2}}\left[\left\langle\vec{\theta}, Z_{h_{1}}(y, \cdot)\right\rangle-\alpha\left\langle\vec{\phi}, Z_{h}(x, \cdot)\right\rangle C-\alpha \eta \vec{b}\right)\right) \\
+f\left(\frac{1}{\sqrt{2}}\left(\left\langle\vec{\theta}, Z_{h_{1}}(y, \cdot)\right\rangle+\alpha\left\langle\vec{\phi}, Z_{h}(x, \cdot)\right\rangle C+\alpha \eta \vec{b}\right)\right) \\
\left.g_{j}\left(\frac{1}{\sqrt{2}}\left[\left\langle\vec{\theta}, Z_{h_{1}}(y, \cdot)\right\rangle-\alpha\left\langle\vec{\phi}, Z_{h}(x, \cdot)\right\rangle C-\alpha \eta \vec{b}\right)\right)\right]
\end{aligned}
$$

Using (2.21) once more we know that the last expression is equal to the last hand side of (3.5) and this completes the proof.

In Theorem 3.5 below we obtain a formula for the generalized conditional convolution with respect to the first argument of the variation of the first variation of functionals from $E_{\sigma}$. 
Theorem 3.5. Let $F$ and $G$ be as in Theorem 2.9 and let $h, h_{1}$ and $h_{2}$ be in $L_{\infty}[0, T]$. Then

$$
\begin{aligned}
& \left(\left(\delta_{h_{1}, h_{2}} F(\cdot \mid w) * \delta_{h_{1}, h_{2}} G(\cdot \mid w)\right)_{\alpha} \| X_{h}\right)(y, \eta) \\
& =\sum_{j=1}^{n} \sum_{l=1}^{n}\left\langle\theta_{j}, Z_{h_{2}}(w, \cdot)\right\rangle\left\langle\theta_{l}, Z_{h_{2}}(w, \cdot)\right\rangle\left(\left(F_{j} * G_{l}\right)_{\alpha} \| X_{h}\right)\left(Z_{h_{1}}(y, \cdot), \eta\right)
\end{aligned}
$$

for all $y, w \in K$ and a.e. $\eta \in \mathbb{R}$.

Proof. Applying the additive distribution properties of the conditional convolution to the expressions given by (2.23) and the corresponding expression for $G$,

$$
\delta_{h_{1}, h_{2}} G(y \mid w)=\sum_{l=1}^{n}\left\langle\theta_{l}, Z_{h_{2}}(w, \cdot)\right\rangle G_{l}\left(Z_{h_{1}}(y, \cdot)\right)
$$

yields equation (3.6) as desired.

We restrict our attention, in this subsequent, to the functions $h_{1}$ and $h_{2}$ either of them is constant on $[0, T]$ rather than to be in $L_{\infty}[0, T]$.

In Theorem 3.6 below we obtain a formula for the generalized conditional convolution product with respect to the second argument of the variation of the first variation of functionals from $E_{\sigma}$.

Theorem 3.6. Let $F$ and $G$ be as in Theorem 2.9 and let $h$, and $h_{1}$ be in $L_{\infty}[0, T]$ and $h_{2}$ be a constant function. Then for a.e. $\eta \in \mathbb{R}$,

$$
\begin{aligned}
& \left(\left(\delta_{h_{1}, h_{2}} F(y \mid \cdot) * \delta_{h_{1}, h_{2}} G(y \mid \cdot)\right)_{\alpha} \| X_{h}\right)(w, \eta)=\frac{1}{2} \delta_{h_{1}, h_{2}} F(y \mid w) \delta_{h_{1}, h_{2}} G(y \mid w) \\
& +\frac{\alpha h_{2} \eta}{2} \sum_{j=1}^{n}\left[\delta_{h_{1}, h_{2}} G(y \mid w) b_{j} F_{j}\left(Z_{h_{1}}(y, \cdot)\right)-\delta_{h_{1}, h_{2}} F(y \mid w) b_{j} G_{j}\left(Z_{h_{1}}(y, \cdot)\right)\right] \\
& -\frac{\alpha^{2} h_{2}^{2}}{2} \sum_{j=1}^{n} \sum_{l=1}^{n}\left(\eta^{2} b_{j} b_{l}+D_{j, l: h}\right) F_{j}\left(Z_{h_{1}}(y, \cdot)\right) G_{l}\left(Z_{h_{1}}(y, \cdot)\right)
\end{aligned}
$$

for all $y$ and $w$ in $K$ with $D_{j, l: h}$ given by equation (3.3). 
Proof. Using the definition of the conditional convolution product, together with equations (2.23) and (3.7), we obtain that

$$
\begin{aligned}
& \left(\left(\delta_{h_{1}, h_{2}} F(y \mid \cdot) * \delta_{h_{1}, h_{2}} G(y \mid \cdot)\right)_{\alpha} \| X_{h}\right)(w, \eta) \\
& =\frac{1}{2} \sum_{j=1}^{n} \sum_{l=1}^{n} F_{j}\left(Z_{h_{1}}(y, \cdot)\right) G_{l}\left(Z_{h_{1}}(y, \cdot)\right) E_{x}\left[\left\{\left\langle\theta_{j} h_{2}, w\right\rangle+\alpha h_{2}\left\langle\theta_{j}-b_{j}, Z_{h}(x, \cdot)\right\rangle\right.\right. \\
& \left.\left.+\alpha h_{2} \eta b_{j}\right\}\left\{\left\langle\theta_{l} h_{2}, w\right\rangle-\alpha h_{2}\left\langle\theta_{l}-b_{l}, Z_{h}(x, \cdot)\right\rangle-\alpha h_{2} \eta b_{l}\right\}\right] .
\end{aligned}
$$

Hence using Lemma 3.1 we see that

$$
\begin{aligned}
& \left(\left(\delta_{h_{1}, h_{2}} F(y \mid \cdot) * \delta_{h_{1}, h_{2}} G(y \mid \cdot)\right)_{\alpha} \| X_{h}\right)(w, \eta) \\
& =\frac{1}{2} \sum_{j=1}^{n} \sum_{l=1}^{n} F_{j}\left(Z_{h_{1}}(y, \cdot)\right) G_{l}\left(Z_{h_{1}}(y, \cdot)\right)\left\{\left\langle\theta_{j} h_{2}, w\right\rangle\left\langle\theta_{l} h_{2}, w\right\rangle\right. \\
& \left.\quad+\alpha h_{2} \eta\left(b_{j}\left\langle\theta_{l} h_{2}, w\right\rangle-b_{l}\left\langle\theta_{j} h_{2}, w\right\rangle\right)-\alpha^{2} h_{2}^{2} \eta^{2} b_{j} b_{l}-\alpha^{2} h_{2}^{2} D_{j, l: h}\right\} .
\end{aligned}
$$

Finally, using equations (2.23) and (3.7) again, it follows that the right hand side of (3.9) equals the right hand side of (3.8) as desired.

In Theorem 3.7 below we get a formula for the generalized conditional integral transform with respect to the first argument of the variation while in Theorem 3.8 we get a formula for the generalized conditional integral transform with respect to the second argument of the variation.

Theorem 3.7. Let $F \in E_{\sigma}$ be given by (2.11) and let $h$ be in $L_{2}[0, T]$, $h_{2}$ be in $L_{\infty}[0, T]$ and $h_{1}$ be a constant function. Then for a.e. $\eta \in \mathbb{R}$,

$$
\mathcal{F}_{\alpha, \beta}\left(\delta_{h_{1}, h_{2}} F(\cdot \mid w) \| X_{h}\right)(y, \eta)=\sum_{j=1}^{n}\left\langle\theta_{j}, Z_{h_{2}}(w, \cdot)\right\rangle \mathcal{F}_{\alpha h_{1}, \beta h_{1}}\left(F_{j} \| X_{h}\right)(y, \eta)
$$

and

$$
\delta_{h_{1}, h_{2}} \mathcal{F}_{\alpha, \beta}\left(F \| X_{h}\right)(\cdot, \eta)(y \mid w)=\beta \sum_{j=1}^{n}\left\langle\theta_{j}, Z_{h_{2}}(w, \cdot)\right\rangle \mathcal{F}_{\alpha, \beta h_{1}}\left(F_{j} \| X_{h}\right)(y, \eta)
$$

for all $y$ and $w$ in $K$. 
Proof. Using the definition of conditional integral transform, equations (2.23) and (3.1), it follows that

$$
\begin{aligned}
& \mathcal{F}_{\alpha, \beta}\left(\delta_{h_{1}, h_{2}} F(\cdot \mid w) \| X_{h}\right)(y, \eta) \\
& =E_{x}\left[\sum_{j=1}^{n}\left\langle\theta_{j}, Z_{h_{2}}(w, \cdot)\right\rangle f_{j}\left(\alpha\left\langle h_{1} \vec{\theta}, Z_{T, \eta}^{\{h, a\}}(x, \cdot)\right\rangle+\beta\left\langle h_{1} \vec{\theta}, y\right\rangle\right)\right] \\
& =\sum_{j=1}^{n}\left\langle\theta_{j}, Z_{h_{2}}(w, \cdot)\right\rangle E_{x}\left[f_{j}\left(\left\langle\vec{\theta}, \alpha h_{1} Z_{T, \eta}^{\{h, a\}}(x, \cdot)+\beta h_{1} y\right\rangle\right)\right] .
\end{aligned}
$$

Furthermore, using the definition of generalized first variation and equation (2.19) we obtain that

$$
\begin{aligned}
& \delta_{h_{1}, h_{2}} \mathcal{F}_{\alpha, \beta}\left(F \| X_{h}\right)(\cdot, \eta)(y \mid w) \\
& =\beta \sum_{j=1}^{n}\left\langle\theta_{j}, Z_{h_{2}}(w, \cdot)\right\rangle E_{x}\left[f_{j}\left(\alpha\left\langle\vec{\phi}, Z_{h}(x, \cdot)\right\rangle C+\alpha \eta \vec{b}+\beta h_{1}\langle\vec{\theta}, y\rangle\right)\right] \\
& =\beta \sum_{j=1}^{n}\left\langle\theta_{j}, Z_{h_{2}}(w, \cdot)\right\rangle \mathcal{F}_{\alpha, \beta h_{1}}\left(F_{j} \| X_{h}\right)(y, \eta)
\end{aligned}
$$

as we wished.

Theorem 3.8. Let $F \in E_{\sigma}$ be given by (2.11) and let $h, h_{1}$ be in $L_{\infty}[0, T]$ and $h_{2}$ be a constant function. Then for a.e. $\eta \in \mathbb{R}$,

$$
\mathcal{F}_{\alpha, \beta}\left(\delta_{h_{1}, h_{2}} F(y \mid \cdot) \| X_{h}\right)(w, \eta)=\beta \delta_{h_{1}, h_{2}} F(y \mid w)+\alpha \eta h_{2} \sum_{j=1}^{n} b_{j} F_{j}\left(Z_{h_{1}}(y, \cdot)\right)
$$

for all $y$ and $w$ in $K$.

Proof. Using the definition of the generalized conditional integral transform, equation (2.23), equation (3.1) and then equation (2.23) again, it 
follows that

$$
\begin{aligned}
& \mathcal{F}_{\alpha, \beta}\left(\delta_{h_{1}, h_{2}} F(y \mid \cdot) \| X_{h}\right)(w, \eta) \\
& =E_{x}\left[\sum_{j=1}^{n}\left\langle\theta_{j} h_{2}, \alpha Z_{T, \eta}^{\{h, a\}}(x, \cdot)+\beta w\right\rangle f_{j}\left(\left\langle\vec{\theta}, Z_{h_{1}}(y, \cdot)\right\rangle\right)\right] \\
& =\sum_{j=1}^{n} f_{j}\left(\left\langle\vec{\theta}, Z_{h_{1}}(y, \cdot)\right\rangle\right) h_{2} E_{x}\left[\beta\left\langle\theta_{j}, w\right\rangle+\alpha\left\langle\theta_{j}-b_{j}, Z_{h}(x, \cdot)\right\rangle+\alpha \eta b_{j}\right] \\
& =\beta \sum_{j=1}^{n}\left\langle\theta_{j}, Z_{h_{2}}(w, \cdot)\right\rangle f_{j}\left(\left\langle\vec{\theta}, Z_{h_{1}}(y, \cdot)\right\rangle\right)+\alpha \eta h_{2} \sum_{j=1}^{n} b_{j} f_{j}\left(\left\langle\vec{\theta}, Z_{h_{1}}(y, \cdot)\right\rangle\right)
\end{aligned}
$$

as we wished.

\section{References}

[1] R.H. Cameron, The first variation of an indefinite Wiener integral, Proc. Amer. Math. Soc. 2 (1951), 914-924.

[2] R.H. Cameron and W.T. Martin, Fourier-Wiener transforms of analytic functionals, Duke Math. J. 12 (1945), 489-507.

[3] R.H. Cameron and D.A. Storvick, An $L_{2}$ analytic Fourier-Feynman transform, Michigan Math. J. 23 (1976), 1-30.

[4] R.H. Cameron and D.A. Storvick, Feynman integral of variations of functionals, Gaussian Random Fields (Nagoya, 1990), Ser. Probab. Statist. 1, World Sci. Publ. 1991, 144-157.

[5] K.S. Chang, B.S. Kim and I. Yoo, Integral transform and convolution of analytic functionals on abstract Wiener spaces, Numer. Funct. Anal. Optim. 21 (2000), 97-105.

[6] S.J. Chang and D. Skoug, Parts formulas involving conditional Feynman integrals, Bull. Aust. Math. Soc. 65 (2002), 353-369.

[7] D.M. Chung, C. Park and D. Skoug, Generalized Feynman integrals via conditional Feynman integrals, Michigan Math. J. 40 (1993), 377-391.

[8] D.M. Chung and D. Skoug, Conditional analytic Feynman integrals and a related Schrödinger integral equation, SIAM J. Math. Anal. 20 (1989), 950-965.

[9] B.A. Fuks, Theory of analytic functions of several complex variables, Amer. Math. Soc., Providence, Rhode Island, 1963.

[10] T. Huffman, C. Park and D. Skoug, Analytic Fourier-Feynman transforms and convolution, Trans. Amer. Math. Soc. 347 (1995), 661-673.

[11] B.J. Kim, B.S. Kim and D. Skoug, Integral transforms, convolution products and first variations, Int. J. Math. Math. Sci. 11 (2004), 579-598.

[12] B.J. Kim, B.S. Kim and D. Skoug, Conditional integral transforms, conditional convolution products and first variations, Panamer. Math. J. 14 (2004), 27-47. 
[13] B.S. Kim and D. Skoug, Integral transforms of functionals in $L_{2}\left(C_{0}[0, T]\right)$, Rocky Mountain J. Math. 33 (2003), 1379 - 1393.

[14] Y.J. Lee, Integral transforms of analytic functions on abstract Wiener spaces, J. Funct. Anal. 47 (1982), 153-164.

[15] C. Park and D. Skoug, Conditional Fourier-Feynman transforms and conditional convolution products, J. Korean Math. Soc. 38 (2001), 61-76.

[16] _ A simple formula for conditional Wiener integrals with applications, Pacific J. Math. 135 (1988), 381-394.

[17] _ A Kac-Feynman integral equation for conditional Wiener integrals, J. Integral Equations Appl. 3 (1991), 411-427.

[18] C. Park, D. Skoug and D. Storvick, Relationships among the first variation, the convolution product, and the Fourier-Feynman transform, Rocky Mountain J. Math. 28 (1998), 1447-1468.

[19] D. Skoug and D. Storvick, A survey of results involving transforms and convolutions in function space, Rocky Mountain J. Math. 34 (2004), 1147-1175.

[20] J. Yeh, Convolution in Fourier-Wiener transform, Pacific J. Math. 15 (1965), $731-738$

[21] _ Inversion of conditional Wiener integral, Pacific J. Math. 59 (1975), 623-638.

[22] I. Yoo, Convolution and the Fourier-Wiener transform on abstract Wiener space, Rocky Mountain J. Math. 25 (1995), 1577-1587.

Department of Mathematics

Daejin University

Pocheon 487-711, Korea

E-mail: bjkim@daejin.ac.kr

School of Liberal Arts

Seoul National University of Science and Technology

Seoul 139-743, Korea

E-mail: mathkbs@seoultech.ac.kr 